\title{
Chemical and Functional Separation of Pro- and Antiangiogenic Components of Danhong Injection in Ischemic or Tumor Vascular Models
}

\section{Shuang He}

Tianjin University of Traditional Chinese Medicine

\section{Rongrong Chen}

Tianjin University of Traditional Chinese Medicine

\section{Li Peng}

Tianjin University of Traditional Chinese Medicine

\section{Zhenzuo Jiang}

Tianjin University of Traditional Chinese Medicine

\section{Haixin Liu}

Tianjin University of Traditional Chinese Medicine

\section{Zihao Chen}

Tianjin University of Traditional Chinese Medicine

\section{Tiechan Zhao}

Tianjin University of Traditional Chinese Medicine

\section{John Orgah}

Tianjin University of Traditional Chinese Medicine

Jie Ren

Tianjin University of Traditional Chinese Medicine

\section{Peng Zhang}

Tianjin University of Traditional Chinese Medicine

\section{Yuefei Wang}

Tianjin University of Traditional Chinese Medicine

\section{Xiumei Gao}

Tianjin University of Traditional Chinese Medicine

Yan Zhu ( $\nabla$ yanzhu.harvard@iCloud.com )

Tianjin University of Traditional Chinese Medicine https://orcid.org/0000-0002-3197-7936

\section{Research}

Keywords: Danhong injection, Angiogenesis, Bi-directional therapy, Proangiogenic component, Antiangiogenic component 
Posted Date: October 28th, 2021

DOl: https://doi.org/10.21203/rs.3.rs-961602/v1

License: (c) (i) This work is licensed under a Creative Commons Attribution 4.0 International License. Read Full License 


\section{Abstract}

Objective: We aimed to investigate the chemical basis and mechanism of angiogenesis regulation by a multicomponent Chinese medicine Danhong injection (DHI).

Methods: A chemical fraction library of DHI was screened and validated for angiogenesis activities by tube formation and migration assays. Mouse ischemic and tumor vascular models were used to verify the angiogenesis effects in vivo. Migration ability of the main monomers of proangiogenic component (PAC) and antiangiogenic component (AAC) in EA.hy 926 cells were determined by migration assay. qPCR analyses were performed to access whether the main monomers of PAC or AAC could affect the expression of angiogenesis-related genes in ECs. Western blotting was used to verify the main monomers PAC and AAC effects on CXCR4 protein expression.

Results: Two chemically-distinct fractions, including promotion and inhibition of angiogenesis, were identified in DHI. PAC enhanced angiogenesis and improved recovery of ischemic limb perfusion while AAC reduced Lewis lung tumor growth in vivo in VEGFR-2-Luc mice. CA and RA upregulated the expression of TSP1 and downregulated the expression of KDR and PECAM genes. CXCR4 expression was significantly decreased by CA and RA, but increased by PAI, consistent with their differential effects on EC migration.

Conclusion: $\mathrm{DH}$ is capable of bi-directional regulation of angiogenesis in a disease-specific manner. The proangiogenesis activity of $\mathrm{DHI}$ promotes ischemic vascular injury repair, whereas the anti-angiogenesis activity inhibits tumor growth. The best pro- and anti-angiogenesis activities are composed of unique chemical combinations that differentially regulate angiogenesis-related gene network.

\section{Introduction}

Angiogenesis is a fundamental process of normal development and various pathophysiology, such as ischemic heart disease, atherosclerosis, limb disease and cancer. (1). Inadequate angiogenesis can lead to ulcer healing disorder and myocardial infarction. Angiogenesis has the potential to rescue early ischemic myocardium and is essential for long-term left ventricular remodeling to prevent heart failure (2, $3)$. In contrast, many pathological conditions, such as cancer and diabetic retinopathy, are characterized by abnormal vascular proliferation. Anti-angiogenic therapy can reprogram the tumor immune microenvironment and prune the blood vessel which is necessary for tumor growth and metastasis (4). Therefore, inhibition of angiogenesis has become a common target of many cancer chemopreventive agents (5). Nowadays, more than 10 approved antiangiogenic tyrosine kinase inhibitors for treating patients with advanced cancers (6). Traditional Chinese medicine (TCM) and other herbal products are a rich source of angiogenesis modulating agents. For example, Ginseng extract has a two-way regulation of angiogenesis, which can promote wound healing and anti-tumor. Those effects were found to be attributable to two opposing ginsenosides Rg1 and Rb1 (7). Our analysis showed that many phytoestrogens are also capable of either promote or inhibit angiogenesis (8). A pharmacological review 
of TCM with angiogenesis showed that tanshinones had the effect of inhibiting angiogenesis, whereas salvianolic acid could promote angiogenesis (9). RA could effectively suppress tumor growth by regulating the secretion of cytokines associated with inflammation and angiogenesis (Cao et al., 2016).

Danhong Injection (DHI) is a proprietary Chinese medicine consisting of extracts from Radix Salviae miltiorrhizae and Flos Carthami tinctorii, has the actions of activating blood circulation and removing stasis. In China, DHI is mainly used for the treatment of cardiovascular and cerebrovascular diseases in the clinic. The pharmacological properties of DHI include anti-inflammatory, antioxidant, anticoagulatory, hypolipidemic effect, antiapoptotic effect, vasodilator, and angiogenesis-promotor (10). We quantified twenty-three primary metabolites and seven polyphenolic acids in DHI (11). Other researchers identified a total of sixty-three compounds in DHI, including thirty-three phenolic acids, six organic acids, six flavonoid O-glycosides, five amino acids, four iridoid glycosides, three nucleosides and two C-glycosyl quinochalcones (12). Our previous studies have shown that DHI increased endothelium-dependent vasorelaxation through the prostacyclin/cyclooxygenase-2 pathway in vivo and in vitro in rat aorta (13). $\mathrm{DHI}$ protected rat cardiomyocyte injury induced by excessive arginine vasopressin and significantly reduced the damage of primary rat neuronal cells and rat cardiomyocytes (14). Studies have shown that DHI promote angiogenesis in ischemic areas $(10,15-21)$. Previous studies by us also shown that DHI promotes endothelial progenitor cell-mediated angiogenesis (He and Guo et al., 2019).

The pharmacological effects of Salvia and safflower are extensive and complex, which shows that DHI can not only play a role in cardiovascular disease but may also have a potential anti-tumor effect. The literature confirms that DHI can promote the apoptosis of hepatoma cells and induce the expression of tumor suppressor gene NDRG2 (22). Therefore, we assumed that DHI might have a potential role as a bidirectional regulation of angiogenesis.

Presently, we mainly carried out a study on the substance basis and mechanism of DHI as a means of regulating angiogenesis by the reverse pharmacology method. After the appropriate separation of DHI, we analyzed the compounds by using modern analytical methods (HPLC and LC-MS), and then each part was screened to determine the active components. We further studied the effective components to clarify its mechanism of action for a better application in the clinic. In vivo experiments were done to verify the effect of pro-angiogenic component (PAC) and anti-angiogenic component (AAC) of DHI. The effective components of the fractions were analyzed and identified by a modern analytical technique. The cell was screened to elucidate the mechanism of angiogenesis at the gene and protein level, and to provide some experimental basis for the clinical application of $\mathrm{DHI}$.

\section{Methods}

\section{Identification of PAC and AAC components by Chromatography}


DHI (product approval number Z20026866) was provided by Shandong Buchang Pharmaceutical Co. Ltd. (Heze, China). The chemical analysis department extracted and separated DHI, and extracted it with dichloromethane and chloroform in sequence according to the polarity. The extract is concentrated under reduced pressure to obtain a dichloromethane partial extract, which is the PAC. The extract is concentrated under reduced pressure to obtain a chloroform partial extract, which is the AAC. As we have previously reported (23), chromatographical identification of PAC and AAC components was performed by an ultra-performance liquid chromatography system (Waters Corp., Milford, MA, USA) equipped with a diode array detector, a column oven, an automatic sampler and a binary gradient solvent pump. We performed chromatography on an ACQUITY UPLC HSS T3 column $(2.1 \times 100 \mathrm{~mm}, 1.8 \mu \mathrm{m})$ at $40^{\circ} \mathrm{C}$. The mobile phase consisted of $0.1 \%$ formic acid aqueous solution (A) and acetonitrile (B). The gradient program was as following: 0 7 min, 3 19\% B; $7 \sim 13 \mathrm{~min}, 19 \% \mathrm{~B} ; 13 \sim 18 \mathrm{~min}, 19 \sim 25 \% \mathrm{~B} ; 18 \sim 25 \mathrm{~min}$, $25 \sim 90 \% \mathrm{~B} ; 25 \sim 30 \mathrm{~min}, 90 \% \mathrm{~B}$. We set the flow rate of the mobile phase to $0.4 \mathrm{~mL} / \mathrm{min}$ and the injection volume was $2 \mu \mathrm{L}$ at the detection wavelengths of 254 and $286 \mathrm{~nm}$.

\section{Cell culture}

EA.hy 926 cells (Shanghai Cell Bank, Type Culture Collection Committee, Chinese Academy of Sciences) were cultured in DMEM (Gibco, USA) supplemented with 10\% fetal bovine serum (FBS) (Gibco, USA), $100 \mathrm{U} / \mathrm{mL}$ penicillin and $100 \mu \mathrm{g} / \mathrm{mL}$ streptomycin (Hyclone, Thermo Scientific, USA) and were grown in an incubator containing $5 \% \mathrm{CO}_{2}$ at $37^{\circ} \mathrm{C}$. When reached the desired confluence, cells were passaged by detaching with $0.25 \%$ trypsin-EDTA (Gibco, USA). Precisely weigh several milligrams of the extract from each layer of the extract of DHI, use DMSO as the solvent, uniformly prepare the mother liquor concentration of $100 \mathrm{mg} / \mathrm{mL}$, and dilute to the working concentration with cell culture medium.

\section{Cell migration assay}

Cell migration was measured in two different assays. EA.hy926 cells were seeded into $96-$ well plates at a density of $2 \times 10^{4}$ cells per well. Cells were stained with $1 \mu \mathrm{g} / \mathrm{mL}$ Hoechst 33342 (Molecular Probes, USA) at $37^{\circ} \mathrm{C}$ for $30 \mathrm{~min}$ after 24 hours of serum deprivation. The cell monolayer was scraped in a straight line to create a "scratch" with a 200 $\mu$ l pipet tip, rinsed twice with PBS to remove the debris and then replaced in $100 \mu$ l experimental DMEM containing $1 \%$ FBS and PAC $(50 \mu \mathrm{g} / \mathrm{mL}), A A C(25 \mu \mathrm{g} / \mathrm{mL})$, or vehicle. The plate was inserted into an incubator at $37^{\circ} \mathrm{C}$ for $12 \mathrm{~h}$. Images were captured at the beginning, $4 \mathrm{~h}, 8 \mathrm{~h}$, and $12 \mathrm{~h}$ after injury using a High Content Screening (HCS) Microplate Reader (Operetta, PerkinElmer, USA) at x10 magnification. The cell migration was quantified using the line selection tool in ImageJ software by tracing the wound margin of two defined positions in each image. By comparing the images from time 0 to the next time point, the distance of each scratch closure was calculated. The oris ${ }^{\text {TM }}$ assay was performed in an optically clear 96-well black skirted fluorescence microplate according to the manufacturer's instruction (24). Briefly, cells are seeded and allowed to attach and spread in the presence of a silicon stopper. Subsequently, the plug was removed and it was found that the central cell-free region was surrounded by monolayer cells and migration can occur. EA.hy926 cells were cultured in DMEM containing $10 \%$ FBS and plated on orisTM cell migration-collagen I coated plates containing cell seeding stoppers. The cells were grown for 16 to 20 hours before the stopper was manually removed. The cells 
were washed and replaced the medium with DMEM containing $1 \% \mathrm{FBS}$, and the indicated concentrations of VEGF, PAC, AAC or vehicle. After incubation with the compound for 12 hours, the media were removed, and the cells were stained with Calcein $A M$ for 30 min at $37^{\circ} \mathrm{C}$. After washing twice with PBS, images were captured on the High Content Screening Microplate Imaging Reader. Three technical replicates were done per experiment, and three independent experiments were performed.

\section{In vitro tube formation assay}

Tube formation assay was performed following a procedure by Michaud (25). 96-well plates were coated with basement membrane matrix (BD Biosciences, USA). EA.hy 926 cells were incubated in a drugcontaining medium at a density of $1.5 \times 10^{4}$ cell/well. After 12 hours of incubation, the cells were stained with $1 \mu \mathrm{M}$ calcein $\mathrm{AM}$ for 30 minutes. Nine fields of view were randomly selected in each well. Imaging was performed using the Operetta HCS System, which analyzes the ability of tube formation by calculating the number of cellular networks. Three technical replicates were done per experiment, and three independent experiments were performed.

\section{Real-time quantitative PCR analysis}

The angiogenesis-related genes were examined by real-time quantitative PCR in cultured EA.hy 926 cells treated with PAC, AAC, or vehicle and ischemic gastrocnemius muscle. Total RNA samples were extracted from ischemic muscle or ECs using TriQuick Reagent (Solarbio, Beijing, China), followed by reverse transcription of the RNA samples into complementary DNA using the Transcriptor First Strand cDNA Synthesis Kit (Roche, Germany) in accordance with the manufacturer's protocol.

The resulting cDNA was used as a template for real-time polymerase chain reaction amplification. SYBR Green Master Mix reagent was used for quantitative PCR, and GAPDH was used as an internal control to quantify the level of angiogenesis-related genes. C1000TM Thermal Cycler Sequence Detection System (BIO-RAD, USA) was used to perform the amplification and analysis. Samples were compared using a relative CT method. The fold increase or decrease was determined relative to a blank control after normalizing to a housekeeping gene using $2^{-\Delta \Delta C T}$, GAPDH.

\section{Ingenuity Pathway Analysis}

Ingenuity Pathway Analysis (IPA) tool (Ingenuity Systems, Redwood City, CA) was used to analyse the interactions between the 33 genes. Since PAC mostly increased the expression of VEGFA and decreased the expression of TIMP3, the potential up- or down-regulated genes were predicted by the Map function of IPA, and since AAC mostly decreased the expression of FGF2 and TGFB2, the potential up- or downregulated genes were predicted by the Map function of IPA .

PA's Map function can be used to predict potential up-regulation or down-regulation of genes, and since AAC mostly reduces the expression of FGF2 and TGFB2, IPA's Map function predicts potential up- or down-regulation of genes.

\section{Preparation of standard and sample solutions}


Preparing the standard stock solutions of sixteen standards of $\mathrm{DHI}$ in $50 \%$ methanol aqueous solution with ultrasonic mixing. For sample stock solution, $0.107 \mathrm{~g}$ PAC and $0.125 \mathrm{~g} \mathrm{AAC}$ were placed in a $25 \mathrm{~mL}$ volumetric flask respectively, with $50 \%$ methanol aqueous solution and methanol volume to the scale, ultrasonically mixed completely, and cleared by centrifugation.

\section{Animals}

Homozygous VEGFR-2-Luc male mice (26) were obtained from three transgenic breeding colonies that were maintained in a specific pathogen-free animal lab of TJAB. Animals were housed in cages in the facility in batches and maintained on a normal diet. All experiments were reviewed and approved by the TJAB Animal Experimental Ethics Committee (TJAB-JY-2011-002) and conducted in accordance with the guidelines for use of animal experiments at Tianjin University of Traditional Chinese Medicine. Before the experiment, the animals were allowed to adapt to the environment for 72 hours in a constant temperature of $22^{\circ} \mathrm{C}$ in a 12-hour light/dark cycled, food and water were freely available.

\section{Murine hind-limb ischemic model}

Mice were anesthetized with isoflurane and unilateral hind-limb ischemia was induced as previously described (He et al., 2016). The entire femoral artery and vein of the right hind limb were exposed, and the exposed vessels were ligated at their proximal and distal ends, both vessels were excised in the middle. The intact perfused contralateral limb of each mouse was used as an internal control. After hind-limb ischemia, saline, simvastatin, DHI, PAC, or AAC were administrated daily for 21 days. The dosage of the PAC per mouse was $13.8 \mathrm{mg} / \mathrm{kg}$, and the dosage of the AAC was $69 \mathrm{mg} / \mathrm{kg}$. The preparation method of PAC and AAC in vivo: first dissolve with $10 \%$ volume of absolute ethanol, then add $90 \%$ volume of normal saline to mix, intraperitoneal injection, once a day. LDPI system was used to perform a continuous, noninvasive assessment of ischemic limb microvascular perfusion. The ratio of perfusion in the ischemic limb to perfusion in the healthy limb was monitored periodically as an indicator of recovery of hind limb perfusion.

\section{In vivo tumor Growth}

VEGFR2-Luc mice were used to establish the Lewis lung carcinoma (LLC) animal model. $100 \mu$ saline containing $2.5 \times 10^{5}$ of LLC cells was injected subcutaneously into the axilla of both forelimbs of the VEGFR2-Luc mice. The day after implantation, the animals were randomized into control, DHI, PAC, and AAC-treatment groups. At 11 days after treatment, protruding tumors were found at the injection sites. Using a caliper, the tumor size was measured every three days. At the end of the treatment, mice were sacrificed, and the tumor was removed and weighted.

\section{In vivo bioluminescent imaging}

IVIS1 Lumina K Series III system (PerkinElmer) was used to provide a real-time, rapid in vivo imaging enabling acquisition of biologically relevant events within milliseconds. Mice were anesthetized with isoflurane and then $150 \mathrm{mg} / \mathrm{kg}$ of D-luciferin (PerkinElmer) was injected intraperitoneally for each mouse. The optical signal intensity of VEGFR-2-luc mice were obtained 5 minutes after D-luciferin administration. 
The regions of interest (ROI) from the displayed images were identified on the ischemic sites or tumor sites using Livinglmage ${ }^{\circledR}$ software and quantified as photons per second $(p / s)$.

\section{Western blotting}

EA.hy 926 cells were treated with different drugs in $10 \mathrm{~cm}$ dishes for 24 hours. The cells were lysed with lysis buffer containing protease inhibitor, then the lysate was transferred to a $1.5 \mathrm{ml}$ centrifuge tube and incubated on ice for 30 minutes with a vortex. Total protein was obtained by centrifugation at $12,000 \times \mathrm{g}$ for 30 minutes at $4^{\circ} \mathrm{C}$, and the protein concentration was measured by a BCA kit. After the addition of the loading buffer, the samples were boiled and separated using sodium dodecyl sulfate-polyacrylamide gel electrophoresis (SDS-PAGE). Proteins were transferred to polyvinylidene difluoride (PVDF) membranes, blocked with $5 \%$ skim milk for 2 hours at room temperature, and then incubated with antibodies against CXCR4 or GAPDH overnight on a $4^{\circ} \mathrm{C}$ shaker. The membranes were washed three times with PBST and then incubated with the secondary antibodies for 1 hour at room temperature in the dark. The membranes were washed three times with PBST, and then the chemiluminescence signals were detected by ECL Plus detection system and exposure to X-ray film to produce bands within the linear range.

\section{Statistical Analysis}

Data analyses were performed with SPSS16.0 statistical software. The results were shown as mean \pm SD. Statistical significance was assessed by unpaired Student's $t$-test or by analysis of variance test for comparison between multiple groups. A P-value of $<0.05$ was taken as statistically significant.

\section{Results}

\section{Identification of the contents composition of PAC and AAC by UPLC-UV}

We have previously constructed a fraction library of $\mathrm{DHI}$ and screened several pharmacological activities $(13,23)$. Here, tube-formation assay was used to screen the angiogenic activity in vascular endothelial cells. We identified a fraction that promoted angiogenic activity (F\#II), but surprisingly, another fraction that inhibited angiogenic activity (F\#III), were assigned as PAC and AAC, respectively. The UPLC-UV chromatograms of PAC and AAC along with the standard, are shown in Fig. 1. And quantitation (total percentage) of the compounds in PAC and AAC are shown in Table 1. 
Table 1

The total percentage of the compounds in PAC and AAC.

\begin{tabular}{|llllllllll|}
\hline & 5-HMF & DDS & PAC & PAl & CA & RA & Sal A & Sal C & Total content (\%) \\
\hline PAC & 6.275 & - & - & 4.986 & - & - & - & 0.0402 & 11.43 \\
AAC & 0.519 & 0.595 & 0.408 & 7.35 & 0.269 & 2.064 & 8.769 & 0.065 & 20.4 \\
\hline
\end{tabular}

PAC and AAC impacted oppositely on endothelial cell angiogenesis in the in vitro tube formation assay

Angiogenesis is the process of forming new blood vessels on existing blood vessel beds by a manner of germination or non-germination through endothelial cell proliferation and migration. It is closely related to many physiological and pathological processes. The classic assay for angiogenesis is to determine the ability of endothelial cells in vitro to form a three-dimensional structure. Our results showed that similar to VEGF ( $50 \mathrm{ng} / \mathrm{mL}$ ), PAC promoted, but AAC inhibited the tube formation ability in vitro compared with the control group (Fig. 3).

\section{PAC and AAC affected endothelial cell migration differently}

Effect of PAC and AAC on endothelial cell migration were determined by a wound-healing assay and Oris $^{\text {TM }}$ Cell Migration Assay. Similar to VEGF $(50 \mathrm{ng} / \mathrm{mL})$ control, PAC significantly increased, but AAC significantly decreased the migration rate of EA.hy 926 cells compared with the control after 12h (Fig. 2).

PAC, but not AAC, improved perfusion of ischemic limb and enhanced angiogenesis in VEGFR-2-luc mice by increasing the expression of FGF-2, CXCR4 and PECAM

Laser Doppler perfusion imaging (LDPI) was performed at different time-points $(6,9,13$, and 16 days after hind-limb ischemia surgery). Before surgery, the blood flow ratio between the two hind limbs in all animals was set to 1.0 immediately after induction of hind limb ischemia, the blood flow ratio between ischemic and contralateral normal non-ischemic limbs was $0.128 \pm 0.009$. Blood flow of saline-treated mice recovered to a ratio of $0.28 \pm 0.07$ after 16 days, whereas in DHI, Simvastatin, or PAC-treated mice, the LDPI ratio was accelerated to $0.62 \pm 0.09,0.60 \pm 0.11$, and $0.44 \pm 0.03$ respectively after 16 days. In addition, as early as 13 days, PAC-treated mice showed a significantly better recovery of the limb perfusion. Thus, the recovery of severely impaired blood-flow in HLI mice was significantly improved by DHI, simvastatin, or PAC treatment. On the other hand, AAC had no such impact in improving the recovery of ischemic limb perfusion (Figs. 4A and 4B).

To further evaluate the effect of PAC and AAC in an in vivo ischemia-induced angiogenesis, we used VEGFR-2-Luc mice in which the expression of luciferase is driven by VEGFR-2 promoter so that angiogenesis can be directly observed by using bioluminescent imaging techniques. 
There was no difference in average bioluminescent intensity between the control group and the treatment group before and after HLI surgery. However, compared to control group, $\mathrm{HLI}$ areas in DHI, simvastatin or PAC treatment groups showed higher bioluminescent intensities after 7 days, suggesting an up-regulation of VEGFR-2 in the ischemic area and a beginning of accelerated blood-flow perfusion recovery. In contrast, $\mathrm{HLI}$ areas in AAC treatment group showed a higher bioluminescent intensity after 11 days (Fig. 4C). Quantitative PCR (qPCR) analyses were performed to access whether PAC or AAC could affect the expression of angiogenesis-related genes in HLI mice. As shown in Fig. 5D, the mRNA levels of FGF-2, CXCR4, and PECAM in PAC-treated group were significantly up-regulated compared with the control group, whereas AAC had no effect on the expression of these genes. Taken together, these results provided further evidence that PAC-treatment promoted recovery of blood flow by increasing the capillary density of the ischemic area.

\section{AAC, not PAC, reduced Lewis lung tumor growth by inhibition of VEGFR2-dependent local angiogenesis}

To examine the inhibitory effect of AAC on tumor growth, we transplanted Lewis lung tumor cells in VEGFR2-Luc mice and measured the in-situ tumor size continually with a caliper for 26 days. The results showed that after 17 days of treatment, the average tumor volumes of the control mice reached 722.89 $\mathrm{mm}^{3}$. AAC treatment reduced tumor volume to $232.29 \mathrm{~mm}^{3}$. In contrast, PAC treatment had no effect on tumor volume. Though the tumor volumes AAC-treated mice increased gradually during the 26 dayobservation periods, the growth trend was not as fast as the control and PAC groups (Fig. $5 \mathrm{C}$ ). The above data indicate that AAC strongly retards Lewis lung tumor growth in vivo. At the end of the 26 daytreatment, we sacrificed the mice, removed the tumors, and weighed them. The weight and size of tumors in control, DHI, PAC, and AAC-treated groups were shown in Figure 5B and C. In order to evaluate the effect of AAC in angiogenesis inhibition, Lewis lung tumor cells were transplanted into VEGFR2-Luc mice, where luciferase expression was driven by the VEGFR2 promoter, allowing direct observation of angiogenesis using in vivo bioluminescence intensity. As shown in Figure 5D, VEGFR2 expression measured in BLI was barely detectable in all groups up to 7 days. However, coincided with the tumor development, bioluminescent intensities increased in control, DHI, and PAC-treated groups 11 days after treatment. In contrast, VEGFR2 expression measured in BLI was significantly delayed in AAC-treated group (Fig. 5D). After 25 days of treatment, the bioluminescent intensities in AAC-treated group remained unchanged while the bioluminescent intensities of control, DHI and PAC-treated groups gradually increased (Fig. 5E). These data suggest that AAC could suppress angiogenesis in vivo.

\section{Confirmation of bi-directional regulation of angiogenesis with main monomers in PAC and AAC}

Migration ability of the main monomers of PAC and AAC in EA.hy926 cells were determined by Oris ${ }^{\text {TM }}$ Cell migration assay. Similar to VEGF, PAI and Sal C significantly increased, while CA significantly decreased, migration rate compared with the control group after $12 \mathrm{~h}$ (Fig. 6A). In order to distinguish the molecular mechanisms of bi-directional regulation of angiogenesis by PAC and AAC, QPCR analyses were 
performed to access whether the main monomers of PAC or AAC could affect the expression of angiogenesis-related genes in ECs. As shown in Figure 6B, PAI decreased the expression of TIMP3 and TSP1; Sal C decreased the expression of TIMP3; CA and RA decreased the expression of KDR and PECAM-1, while increased the expression of TSP1. We used western blotting to independently verify the PAC and AAC effects on angiogenesis-related protein expression. As shown in Figure 6C, CXCR4 expression was significantly decreased by CA and RA, but increased by PAI, consistent with their differential effects on EC migration (Fig. 6A).

\section{Discussion}

Our previous experiments have systematically investigated the efficacy and mechanism of DHI in angiogenesis and vascular injury repair. This experiment is to further explore the material basis of DHI on angiogenesis regulation following the previous work. Using reverse pharmacology, DHI chemical fractions were screened by in vitro angiogenesis assay and were found to contain not only a proangiogenic component (PAC) but also an antiangiogenic component (AAC). Subsequently, the pharmacodynamics experiments of PAC and AAC on angiogenesis were carried out in hind-limb ischemia model, and tumor model, which confirmed that PAC could promote, and AAC could inhibit angiogenesis. To further study the material basis, the active constituents of PAC and AAC were isolated and screened by functional experiments. We found that PAI and Sal $\mathrm{C}$ played a major role in promoting angiogenesis, while $\mathrm{CA}$ and PA had the effect of inhibiting angiogenesis.

TIMP3 is a tissue inhibitor of metalloproteinases (MMPs), which downregulates the activity of MMPs to degrade extracellular matrix and inhibit angiogenesis $(27,28)$. Angiogenesis inhibitor TSP1, the platelet reactive protein 1 , is one of the members of the extracellular matrix protein family, which inhibits the degradation of ECM and affect the migration and survival of endothelial cells (29). KDR is an endothelial cell growth factor receptor and is a classic angiogenic receptor (30). PECAM-1 is an endothelial cell adhesion molecule that promotes adhesion between endothelial cells to promote angiogenesis (31). We found that PAI significantly down-regulated the expression of TIMP3 and TSP1 gene, and Sal C significantly down-regulated the expression of TIMP3 gene. On the other hand, CA and RA inhibited the expression of KDR and PECAM-1 genes and promoted the expression of TSP1 gene. Therefore, it can be concluded that the active components in $\mathrm{DHI}$ regulate angiogenesis process mainly by affecting the proliferation, migration, adhesion and degradation of the extracellular matrix of endothelial cells.

In addition, the active compounds in PAC or AAC can regulate the TSP1 expression, indicating that TSP1 is a common target for the bi-directional regulation of angiogenesis by DHI. In addition, PAI and CA also regulate CXCR4 protein expression in a bi-directional manner. CXCR4 is a specific receptor of chemokine SDF-1, which mediates the migration and homing of hematopoietic stem cells and can promote the secretion of VEGF (32). As a G protein-coupled receptor, CXCR4 is important for many cellular functions including migration, mobilization and homing (33) and plays an important role in enhancing angiogenesis in vivo (34). 
One of the challenging tasks of modernization of Chinese herbal medicine is to reveal its complex pharmacological basis and its compatibility mechanism (35). Attentions on the effective combinatorial mixtures are increasing in researching of drug discovery and development (36). Further development of DHI will be based on the traditional Chinese prescription compatibility theory, guided by the systematic scientific thoughts to understand the effective combination of the components for different disease treatment. For example, we have recently identified a combination of four DHI components that are necessary and sufficient for maximum angiogenesis ability.

Combination components exploit the chances for better efficacy and decreased toxicity (37). It is an effective method for developing new modern drugs based on the combination of effective monomers in TCM with exact clinical efficacy.

This present work only examined four monomers (PAl, Sal C, CA, and RA) individually, but the overall impact of PAC or AAC are likely contributed by combinations of multiple components. Therefore, further experiments are needed to clarify the optimal monomer combination that promotes or inhibit angiogenesis. It is also possible that other unidentified compounds exist that potentially contribute to promoting or inhibiting angiogenesis. In DHI, a total of sixty-three compounds have been identified and characterized so far (38). Other monomers in PAC and AAC that promote or inhibit angiogenesis remain to be identified.

Many pro-angiogenic and anti-angiogenic molecules have been identified, and some have been developed into drugs to treat cardiovascular disease and cancer, respectively. However, we showed that the widely used cardiovascular herbal remedy, DHI, has intrinsic pro- and anti-angiogenesis dual activities. Moreover, the bidirectional activities are defined by different yet overlapping combinations of multiple molecules in DHI. Although the clinical significance of this finding has yet to be determined, it would imply that caution shall be called when $\mathrm{DHI}$ is prescribed for cardiovascular disease patients with cancer risk. Further basic science and clinical investigations are necessary to reveal the benefits and shortcomings of this "double-sided sward" in the age of precision medicine.

\section{Conclusion}

We conclude that DHI, a widely used compound Chinese patent medicine, contained both pro- and antiangiogenesis components that compose different chemical combinations. The pro-angiogenesis component can be capable of promoting ischemic vascular injury repair, whereas the anti-angiogenesis components inhibited tumor growth. A key mechanism involved in the promotion of angiogenesis is associated with PAl-mediated TIMP and TSP1 downregulation and CXCR4 upregulation, as well as Sal Cmediated TIMP3 downregulation. We showed for the first time that DHI mechanism of angiogenesis inhibition is associated at least in part with CA/RA-mediated downregulation of KDR, PECAM-1, and CXCR4.

\section{Abbreviations}


HLI

hind-limb ischemia

BLI

Bioluminescence imaging

PAC

pro-angiogenic components

AAC

anti-angiogenic components

5-HMF

5-hydroxymethyl-2-furfural

DSS

Danshensu

PAC

protocatechuic acid

PAl

protocatechuic aldehyde

HSYA

hydroxysafflor yellow $A$

CA

caffeic acid

RA

rosmarinic acid

LA

lithospermic acid

Sal B

salvianolic acid $B$

Sal A

salvianolic acid $A$

Sal C

salvianolic acid $\mathrm{C}$

\section{Declarations}

Ethics approval and consent to participate】All experiments were reviewed and approved by the TJAB Animal Experimental Ethics Committee (TJAB-JY-2011-002) and conducted in accordance with the guidelines for use of animal experiments at Tianjin University of Traditional Chinese Medicine.

Consent for publication $\square$ Not applicable

Availability of data and material[The datasets during and/or analysed during the current study available from the corresponding author on reasonable request. 
Competing interests[The authors declare that they have no competing interests

Funding $[T h i s$ study was supported by the grants from National Natural Science Foundation of China (81873037), the National Major Science and Technology Projects of China (2018ZX01031301) and National Key R\&D Program of China (2018YFC1704500).

Authors' contributions $\square Y Z$ conceived and organized the study; SH, RC, LP, ZJ, HL, TZ and JR performed the experiments; $\mathrm{SH}, \mathrm{RC}, \mathrm{TZ}$ and ZC performed the statistical analysis; $\mathrm{PZ}, \mathrm{YW}$ and $\mathrm{XG}$ helped with the design of the study and interpretation of results; $\mathrm{YZ}$ and $\mathrm{SH}$ wrote the first draft of the manuscript; JO wrote sections of the manuscript. All authors reviewed and approved the manuscript.

Acknowledgements $\square$ Not applicable

\section{References}

1. Rivera LB, Bergers G. Angiogenesis. Targeting vascular sprouts. Science. 2014;344(6191):1449-50.

2. Shah AM, Mann DL. In search of new therapeutic targets and strategies for heart failure: recent advances in basic science. Lancet. 2011;378(9792):704-12.

3. van der Laan AM, Piek JJ, van Royen N. Targeting angiogenesis to restore the microcirculation after reperfused MI. Nature reviews Cardiology. 2009;6(8):515-23.

4. Yi M, Jiao D, Qin S, Chu Q, Wu K, Li A. Synergistic effect of immune checkpoint blockade and antiangiogenesis in cancer treatment. Mol Cancer. 2019;18(1):60.

5. Albini A, Tosetti F, Benelli R, Noonan DM. Tumor inflammatory angiogenesis and its chemoprevention. Cancer research. 2005;65(23):10637-41.

6. Qin S, Li A, Yi M, Yu S, Zhang M, Wu K. Recent advances on anti-angiogenesis receptor tyrosine kinase inhibitors in cancer therapy. J Hematol Oncol. 2019;12(1):27.

7. Sengupta S, Toh SA, Sellers LA, Skepper JN, Koolwijk P, Leung HW, et al. Modulating angiogenesis: the yin and the yang in ginseng. Circulation. 2004;110(10):1219-25.

8. Liu HX, Wang Y, Lu Q, Yang MZ, Fan GW, Karas RH, et al. Bidirectional regulation of angiogenesis by phytoestrogens through estrogen receptor-mediated signaling networks. Chinese journal of natural medicines. 2016;14(4):241-54.

9. Chen RG, Xu H, He Y, Zhu S. Y. Research progress on pro- or anti-angiogenic effects of Chinese materia medica fomulas and their active components. Chinese Traditional Herbal Drugs. 2013;44(23):3413-21.

10. Feng X, Li Y, Wang Y, Li L, Little PJ, Xu SW, et al. Danhong injection in cardiovascular and cerebrovascular diseases: Pharmacological actions, molecular mechanisms, and therapeutic potential. Pharmacological research. 2019;139:62-75.

11. Jiang $M$, Jiao $Y$, Wang $Y, X u L$, Wang $M$, Zhao B, et al. Quantitative profiling of polar metabolites in herbal medicine injections for multivariate statistical evaluation based on independence principal 
component analysis. PloS one. 2014;9(8):e105412.

12. Zhang QQ, Dong X, Liu XG, Gao W, Li P, Yang H. Rapid separation and identification of multiple constituents in Danhong Injection by ultra-high performance liquid chromatography coupled to electrospray ionization quadrupole time-of-flight tandem mass spectrometry. Chinese journal of natural medicines. 2016;14(2):147-60.

13. Wang D, Fan G, Wang Y, Liu H, Wang B, Dong J, et al. Vascular reactivity screen of Chinese medicine danhong injection identifies Danshensu as a NO-independent but PGI2-mediated relaxation factor. J Cardiovasc Pharmacol. 2013;62(5):457-65.

14. Yang M, Orgah J, Zhu J, Fan G, Han J, Wang X, et al. Danhong injection attenuates cardiac injury induced by ischemic and reperfused neuronal cells through regulating arginine vasopressin expression and secretion. Brain research. 2016;1642:516-23.

15. Chen J, Wei J, Huang Y, Ma Y, Ni J, Li M, et al. Danhong Injection Enhances the Therapeutic Efficacy of Mesenchymal Stem Cells in Myocardial Infarction by Promoting Angiogenesis. Frontiers in physiology. 2018;9:991.

16. Li JK, Wang C, Gong HD, Li HZ. Coagulation in hindbrain membrane meningioma patients treated with different injections using acute hypervolemic hemodilution. J Biol Regul Homeost Agents. 2017;31(4):991-6.

17. Wan J, Wan H, Yang R, Wan H, Yang J, He Y, et al. Protective effect of Danhong Injection combined with Naoxintong Capsule on cerebral ischemia-reperfusion injury in rats. J Ethnopharmacol. 2018;211:348-57.

18. He S, Zhao T, Guo H, Meng Y, Qin G, Goukassian DA, et al. Coordinated Activation of VEGF/VEGFR-2 and PPARdelta Pathways by a Multi-Component Chinese Medicine DHI Accelerated Recovery from Peripheral Arterial Disease in Type 2 Diabetic Mice. PLoS One. 2016;11(12):e0167305.

19. Chen J, Cao W, Asare PF, Lv M, Zhu Y, Li L, et al. Amelioration of cardiac dysfunction and ventricular remodeling after myocardial infarction by danhong injection are critically contributed by anti-TGFbeta-mediated fibrosis and angiogenesis mechanisms. J Ethnopharmacol. 2016;194:559-70.

20. Wu F, He Z, Ding R, Huang Z, Jiang Q, Cui H, et al. Danhong Promotes Angiogenesis in Diabetic Mice after Critical Limb Ischemia by Activation of CSE-H 2 S-VEGF Axis. Evidence-based complementary and alternative medicine. eCAM. 2015;2015:276263.

21. He ZQ, Ji RZ, Wang X, Liang C, Wu ZG. [Effect of advanced glycation end products on the function and angiogenesis of adipose tissue-derived stem cells and the protective effect of danhong injection: an experimental study]. Zhongguo Zhong xi yi jie he za zhi Zhongguo Zhongxiyi jiehe zazhi =. Chinese journal of integrated traditional Western medicine. 2014;34(7):839-45.

22. Zheng JL, Shi Q, Wang H, Zhao K, Ding G, Ren J. Q. Danhong injection enhances apoptosis index and NDRG2 expression in hepatocellular carcinoma cells. Journal of Shanxi Medical University. 2010;41(12):1009-12.

23. Zhao T, Chang L, Zhang B, Lu M, Wang X, Orgah JO, et al. Specific Combination of Salvianolic Acids As Core Active Ingredients of Danhong Injection for Treatment of Arterial Thrombosis and Its Derived 
Dry Gangrene. Front Pharmacol. 2017;8:361.

24. Fu J, Yu J, Chen J, Xu H, Luo Y, Lu H. In vitro inhibitory properties of sesquiterpenes from Chloranthus serratus on cell motility via down-regulation of LIMK1 activation in human breast cancer. Phytomedicine: international journal of phytotherapy phytopharmacology. 2018;49:23-31.

25. Michaud SE, Menard C, Guy LG, Gennaro G, Rivard A. Inhibition of hypoxia-induced angiogenesis by cigarette smoke exposure: impairment of the HIF-1alpha/VEGF pathway. FASEB journal: official publication of the Federation of American Societies for Experimental Biology. 2003;17(9):1150-2.

26. Zhang N, Fang Z, Contag PR, Purchio AF, West DB. Tracking angiogenesis induced by skin wounding and contact hypersensitivity using a Vegfr2-luciferase transgenic mouse. Blood. 2004;103(2):61726.

27. Basu R, Lee J, Morton JS, Takawale A, Fan D, Kandalam V, et al. TIMP3 is the primary TIMP to regulate agonist-induced vascular remodelling and hypertension. Cardiovascular research. 2013;98(3):360-71.

28. Qi JH, Anand-Apte B. Tissue inhibitor of metalloproteinase-3 (TIMP3) promotes endothelial apoptosis via a caspase-independent mechanism. Apoptosis: an international journal on programmed cell death. 2015;20(4):523-34.

29. Bienes-Martinez R, Ordonez A, Feijoo-Cuaresma M, Corral-Escariz M, Mateo G, Stenina O, et al. Autocrine stimulation of clear-cell renal carcinoma cell migration in hypoxia via HIF-independent suppression of thrombospondin-1. Scientific reports. 2012;2:788.

30. Carmeliet P. Mechanisms of angiogenesis and arteriogenesis. Nature medicine. 2000;6(4):389-95.

31. Park S, Sorenson CM, Sheibani N. PECAM-1 isoforms, eNOS and endoglin axis in regulation of angiogenesis. Clinical science. 2015;129(3):217-34.

32. Kawakami Y, li M, Matsumoto T, Kuroda R, Kuroda T, Kwon SM, et al. SDF-1/CXCR4 axis in Tie2lineage cells including endothelial progenitor cells contributes to bone fracture healing. Journal of bone mineral research: the official journal of the American Society for Bone Mineral Research. 2015;30(1):95-105.

33. Yamaguchi J, Kusano KF, Masuo O, Kawamoto A, Silver M, Murasawa S, et al. Stromal cell-derived factor-1 effects on ex vivo expanded endothelial progenitor cell recruitment for ischemic neovascularization. Circulation. 2003;107(9):1322-8.

34. Chen L, Wu F, Xia WH, Zhang YY, Xu SY, Cheng F, et al. CXCR4 gene transfer contributes to in vivo reendothelialization capacity of endothelial progenitor cells. Cardiovascular research. 2010;88(3):462-70.

35. Li F, Fan XX, Chu C, Zhang Y, Kou JP, Yu BY. A Strategy for Optimizing the Combination of Active Components Based on Chinese Medicinal Formula Sheng-Mai-San for Myocardial Ischemia. Cellular physiology and biochemistry: international journal of experimental cellular physiology, biochemistry, and pharmacology. 2018;45(4):1455-71.

36. Lu JJ, Pan W, Hu YJ, Wang YT. Multi-target drugs: the trend of drug research and development. PloS one. 2012;7(6):e40262. 
37. Foucquier J, Guedj M. Analysis of drug combinations: current methodological landscape. Pharmacol Res Perspect. 2015;3(3):e00149.

38. Zhang QQ, Dong X, Liu XG, Gao W, Li P, Yang H. Rapid separation and identification of multiple constituents in Danhong Injection by ultra-high performance liquid chromatography coupled to electrospray ionization quadrupole time-of-flight tandem mass spectrometry. Chinese journal of natural medicines. 2016;14(2):147-60.

\section{Figures}

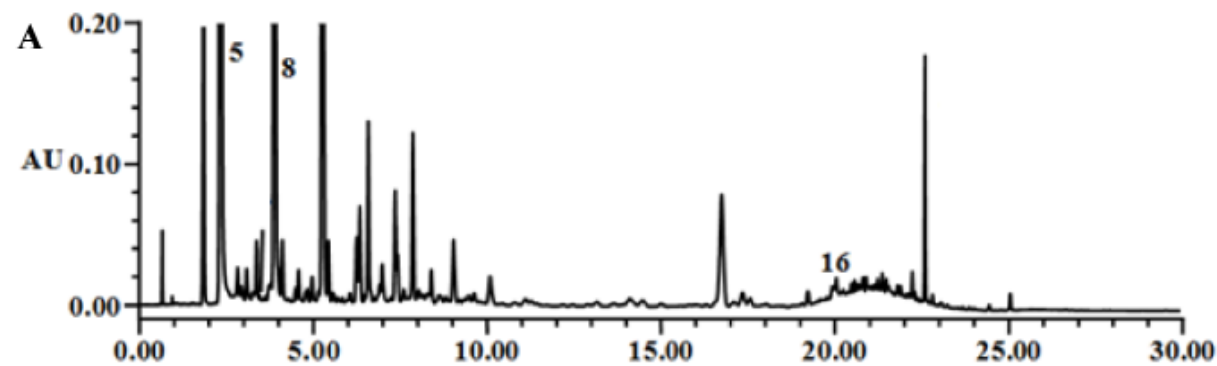

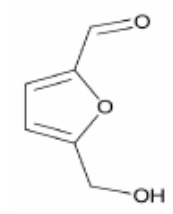

5-HMF

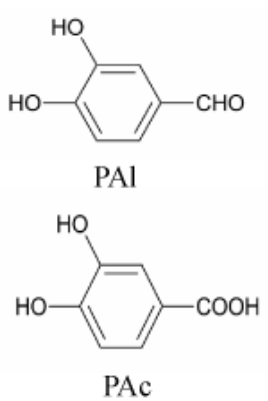

PAc

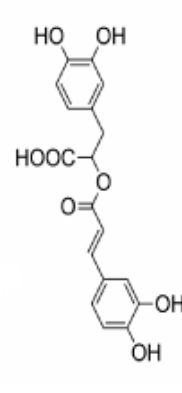

PA

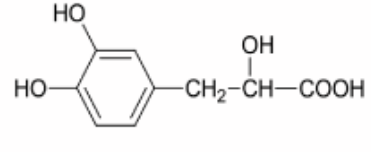

DSS

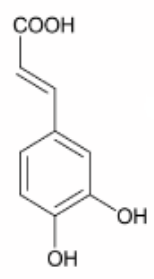

CA

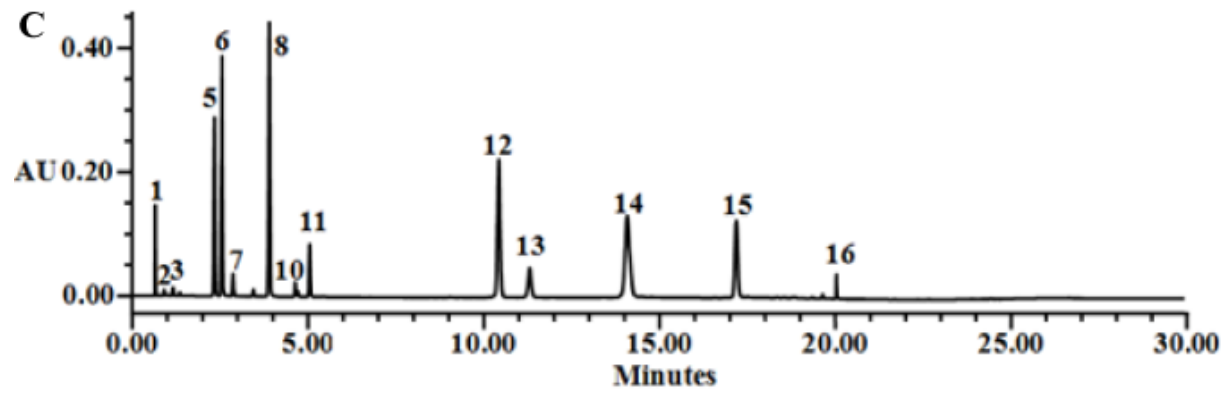

\section{Figure 1}

UPLC-UV chromatograms of (A) PAC, (B) AAC, (C) Standard mixture. Peaks: 1. cytidine, 2. uridine, 3. adenosine, 5. 5-hydroxymethyl-2-furfural (5-HMF), 6. Danshensu (DSS), 7. protocatechuic acid (PAC), 8. protocatechuic aldehyde (PAI), 10. hydroxysafflor yellow A (HSYA), 11. caffeic acid (CA), 12. rosmarinic acid (RA), 13. lithospermic acid (LA), 14. salvianolic acid B (Sal B), 15. salvianolic acid A (Sal A) and 16. salvianolic acid C (Sal C). 
A

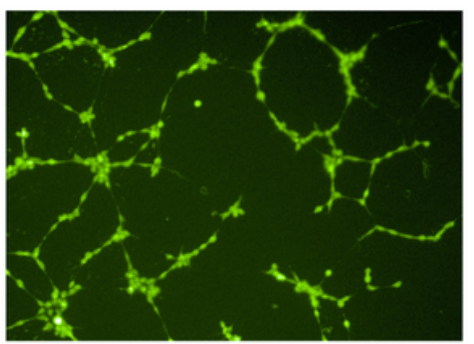

Control

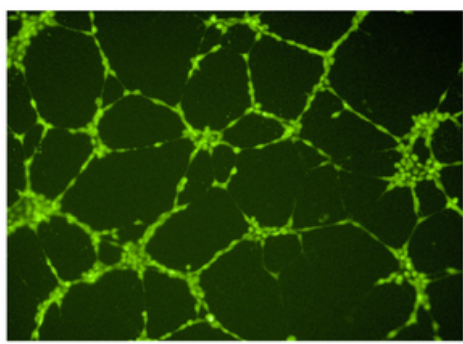

VEGF

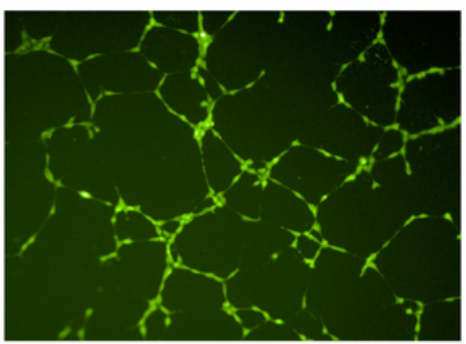

PAC

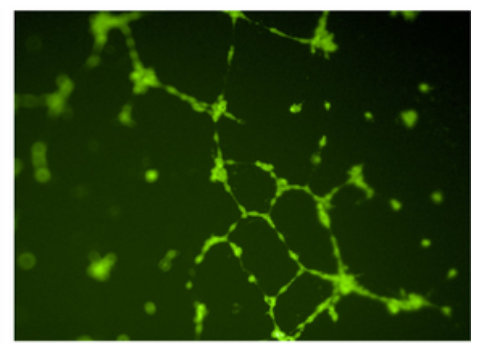

AAC

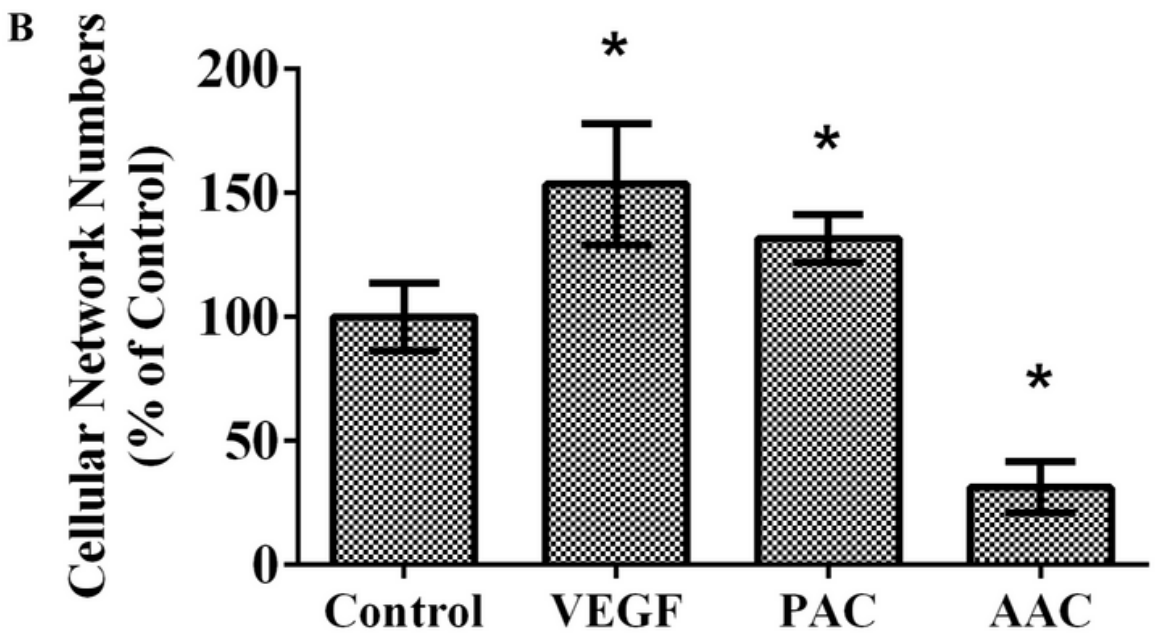

Figure 2

PAC and AAC affected oppositely on endothelial cell angiogenesis in tube formation assay. (A) Representative microscopy showing tube formation of EA.hy926 cells. (B) Quantitation of in vitro tube formation activity. After incubating for $12 \mathrm{~h}$, numbers of the cellular network were counted in multiple ( $\mathrm{n}=$ 3 ) views and averaged percentage was calculated. VEGF and PAC promoted tube formation, whereas AAC inhibited tube formation in EA.hy926 cells. Data are presented as mean $\pm S D$. $n=3 .{ }^{*} P<0.05$, compared with the control group.

\section{Figure 3}

PAC and AAC affected endothelial cell migration differently. (A) Representative images of the woundhealing assay in EA.hy926 cells. (B) Quantitation of the migration distance. PAC or VEGF promoted cell migration while AAC inhibited the cell migration after $12 \mathrm{~h}$. (C) Representative images of the Oris ${ }^{\mathrm{TM}}$ Cell Migration Assay in EA.hy926 cells. (D) Analysis of ECs-covered area after $12 \mathrm{~h}$ incubation or immediately at the stopper removal (pre-migration, 0 hour). The same location, for all wells was set-aside as detection zone (Green Square), and the cell inhabited area was determined under the indicated conditions. VEGF or PAC promotes cell migration while AAC inhibited the cell migration after $12 \mathrm{~h}$. AAC also reversed the cell migration effect of VEGF, leading to an ultimate inhibition of endothelial cell migration. $n=3$. Data are presented as mean $\pm S D$. ${ }^{*} P<0.05$, compared with the control group. 


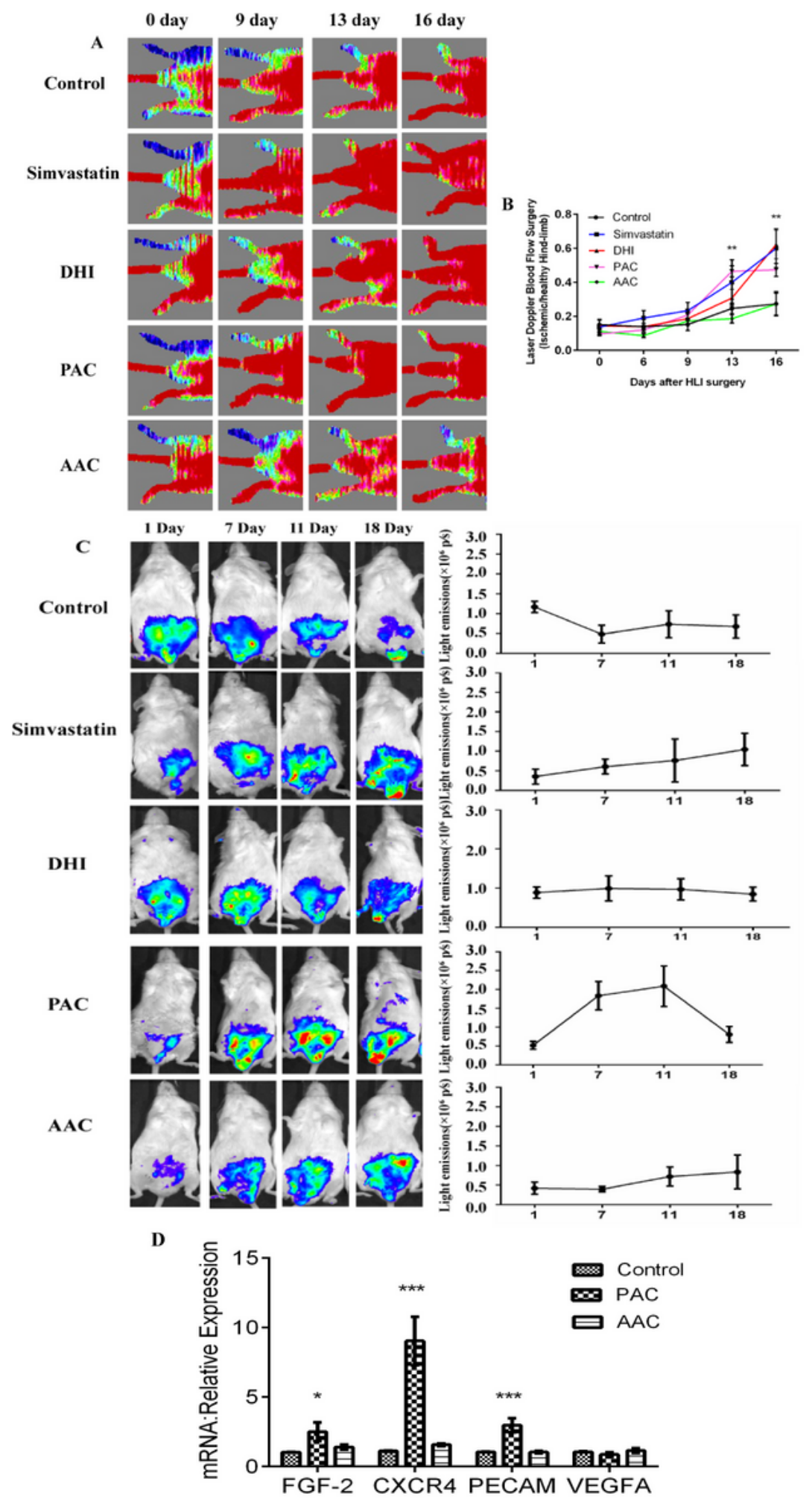

Figure 4

PAC improved perfusion of ischemic limbs and enhanced local angiogenesis. (A) Representative images of laser Doppler perfusion analysis. (B)We calculated the ratio of the ischemic side to the non-ischemic side as the average hindlimb blood flow recovery. PAC significantly improved perfusion recovery on day 13 after $\mathrm{HLI}$ surgery. DHI or simvastatin significantly improved perfusion recovery on day 16 after HLI surgery. ${ }^{*} \mathrm{P}<0.01$ vs. control group. (C) Representative bioluminescent images (left panels) and 
quantitation (right panels) of VEGFR-2-Luc mice after HLI were obtained at 1, 7, 11, and 18 days in control, simvastatin, DHI, PAC, or AAC-treated groups. Regions of interest (ROI) from displayed images were identified on the $\mathrm{HLI}$ sites and quantified as photons per second $(\mathrm{p} / \mathrm{s})$. Only in the hind limb ischemia model, the simvastatin-positive drug group consisted of three mice, and the remaining groups consisted of five mice. (D) Quantitative PCR showed that only PAC increased the expression of FGF-2, CXCR4, and PECAM in ischemic gastrocnemius muscle. ${ }^{\star} P<0.05$, $* \star * P<0.001$ vs. control group. Data are mean \pm SEM. We performed the experiments in two copies and repeated at least three times to assure the reproducibility. 

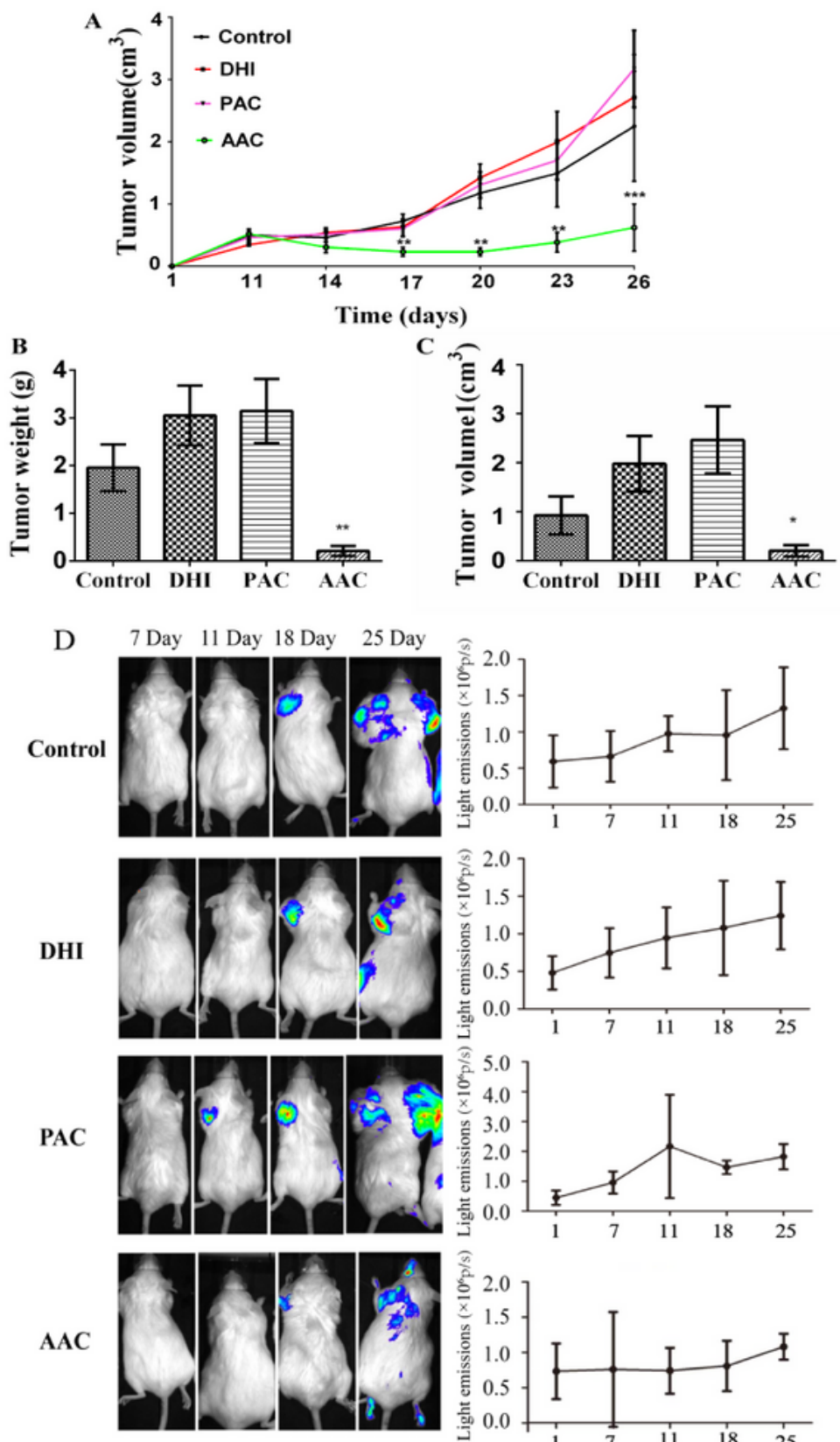

$\mathrm{E}$
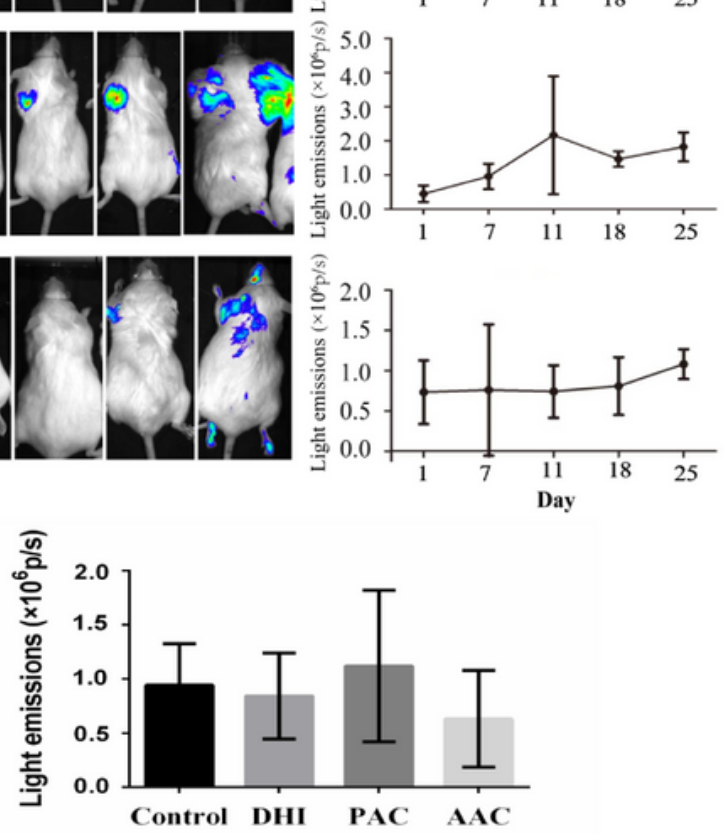

Figure 5

AAC reduced Lewis lung tumor growth. Tumor size was continuously monitored by caliper from the day of tumor cell transplant up to 26 days. (A) Tumor volume curve of saline, DHI, PAC, or AAC-treated mice. (B) Weight of transplanted tumors in each treatment group at day 26. (C) Average tumor volume in each treatment group at day 26. (D) The bioluminescent intensities of the saline, DHI, PAC, or AAC-treated VEGFR2-Luc mice model of tumor was dynamically measured. Regions of interest (ROI) from displayed 
images were identified on the tumor sites and quantified as photons per second $(p / s)$. (E) The bioluminescent intensities of each group on day 25 after treatment. Data are all shown as mean \pm SEM. $n$ $=5$ in each group. ${ }^{*} \mathrm{P}<0.05,{ }^{*} \mathrm{P}<0.01, * \star * \mathrm{P}<0.001$, as compared with control.

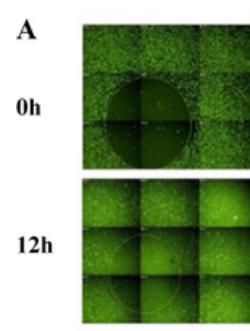

Control

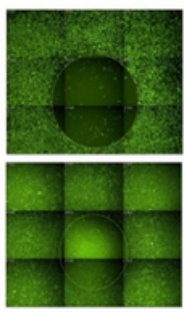

VEGF

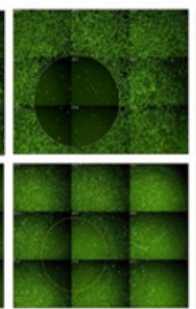

PAI

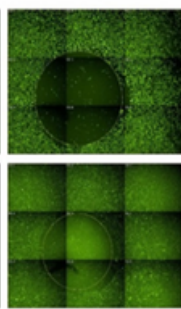

$\mathrm{SaC}$

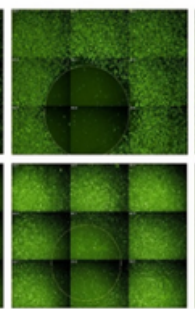

CA

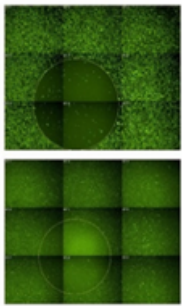

RA
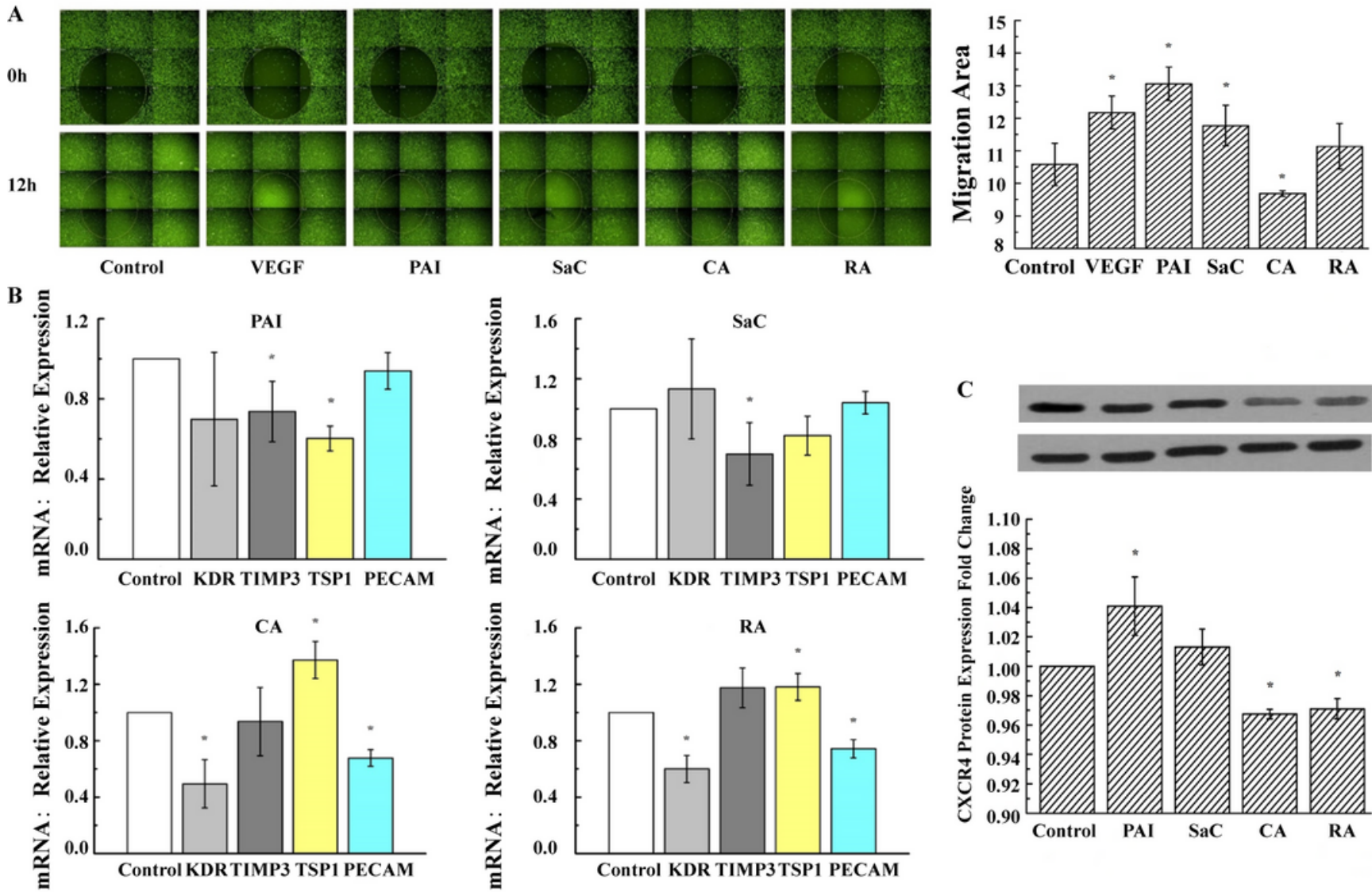
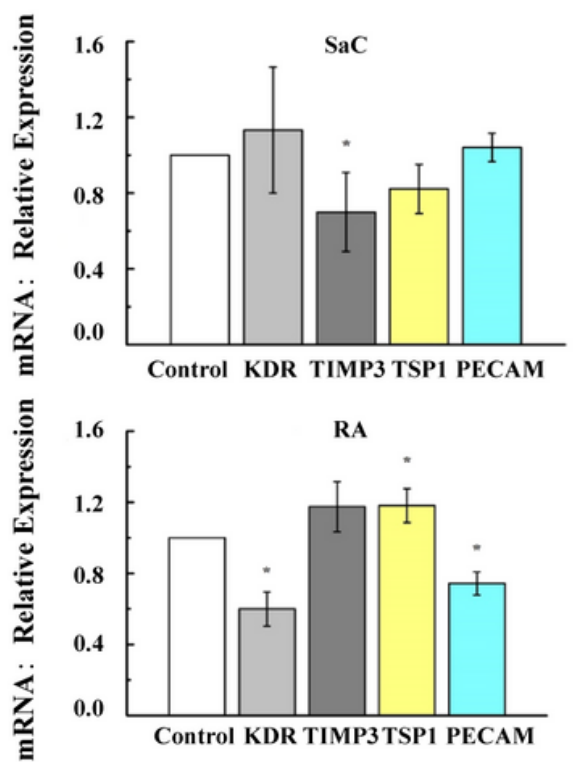

C

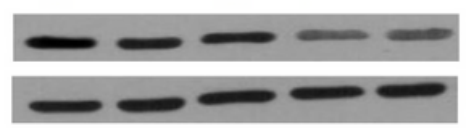

빵
플 1.10
ㄹ 1.08
흥 1.06 .10
.08
.06

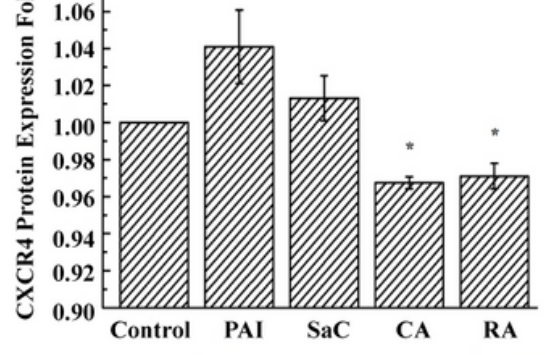

Figure 6

The main monomers of PAC and AAC had a bi-directional regulation of angiogenesis. (A) Representative images of the Oris ${ }^{\text {TM }}$ Cell Migration Assay in EA.hy 926 cells. The EC-covered area was analyzed immediately following stopper removal (pre-migration, $0 \mathrm{~h}$ ) or after drug treatment for 12 hours. A detection area (Green Square) was defined in the same location for all wells and the area covered with cells determined under the indicated conditions. VEGF, PAI or Sal C promoted, while CA inhibited, the cell migration after 12h. (B) Quantitative PCR showing the expression of KDR, TIMP3, TSP1, and PECAM-1 in cells treated with PAI, Sal C, CA, or RA in ECs. (C) Representative Western blot analysis of CXCR4 expression (top) and densitometric quantitation (bottom). Compared with control, CXCR4 expression was significantly increased by PAl treatment but significantly decreased by CA and RA treatment. GAPDH was used as a loading control. ${ }^{*} P<0.05$ vs. control group. $n=3$. Data are presented as mean $\pm S E M$. 